\title{
In-flight calibration of the Hot Ion Analyser on board Cluster
}

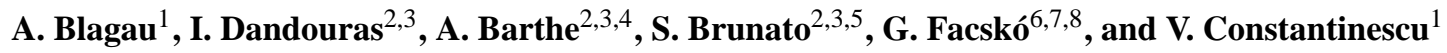 \\ ${ }^{1}$ Institute for Space Sciences, Bucharest, Romania \\ ${ }^{2}$ Institut de Recherche en Astrophysique et Planétologie, Université de Toulouse, Toulouse, France \\ ${ }^{3}$ CNRS, Institut de Recherche en Astrophysique et Planétologie, Toulouse, France \\ ${ }^{4}$ AKKA Technologies, Toulouse, France \\ ${ }^{5}$ Noveltis, Toulouse, France \\ ${ }^{6}$ Laboratoire de Physique et Chimie de l'Environnement et de l'Espace, Orléans, France \\ ${ }^{7}$ Geodetic and Geophysical Institute, Research Centre for Astronomy and Earth Sciences, HAS, Sopron, Hungary \\ ${ }^{8}$ now at: Finnish Meteorological Institute, Helsinki, Finland
}

Correspondence to: A. Blagau (blagau@spacescience.ro)

Received: 23 March 2013 - Published in Geosci. Instrum. Method. Data Syst. Discuss.: 26 July 2013

Revised: 19 February 2014 - Accepted: 20 March 2014 - Published: 23 April 2014

\begin{abstract}
The Hot Ion Analyser (HIA), part of the Cluster Ion Spectrometry experiment, has the objective to measure the three-dimensional velocity distributions of ions. Due to a variety of factors (exposure to radiation, detector fatigue and aging, changes in the operating parameters, etc.), the particles' detection efficiency changes over time, prompting for continuous in-flight calibration. This is achieved by comparing the HIA data with the data provided by the WHISPER (Waves of HIgh frequency and Sounder for Probing of Electron density by Relaxation) experiment on magnetosheath intervals, for the high-sensitivity section of the instrument, or solar wind intervals, for the low-sensitivity section. The paper presents in detail the in-flight calibration methodology, reports on the work carried out for calibrating HIA and discusses plans to extend this activity in order to ensure the instrument's highest data accuracy.
\end{abstract}

\section{Introduction}

The Hot Ion Analyser (HIA) and the COmposition and DIstribution Function (CODIF) analyzer are the two sensors of the Cluster Ion Spectrometry (CIS) experiment (Rème et al., 2001) on board Cluster, having the objective to measure the three-dimensional velocity distributions of ions. As a major difference from CODIF, the HIA instrument does not provide mass resolution; however, HIA offers other important advantages, like higher detection efficiency, better angular and en- ergy resolution, faster electronics capable to handle higher count rates, etc.

The HIA detection system is based on micro-channel plate (MCP) technology. The instrument efficiency has been determined on ground through extensive preflight calibrations. However, due to various reasons, like MCP gain fatigue and aging (Prince and Cross, 1971) or because of the penetrating radiation (in the radiation belts or from cosmic ray bombardment), the detector efficiency changes in the course of the mission, requiring periodic in-flight calibration. An in-flight calibration is needed as well whenever the HIA operating point is changed by commands from ground. The multipoint character of Cluster and its complex payload made it possible to asses the HIA's in-flight performance at an unprecedented level of accuracy.

The calibration methodology to be presented in this paper was developed by taking advantage of the large crosscalibration effort carried on in the framework of ESA's Cluster Active Archive (CAA) program. Before the CAA initiative, the resources allocated to this activity were relatively small when compared with the complexity of the work; also, the less accurate Cluster Prime Parameter data set has been used as a reference for the total electron density (Vallat, 2001).

The CIS experiment is prepared by an international consortium, under the principal responsibility of Institut de Recherche en Astrophysique et Planétologie (IRAP) in Toulouse (formerly Centre d'Etude Spatiale des 
Rayonnements). Since 2009, the Institute for Space Sciences in Bucharest assumed a key role in HIA's in-flight calibration, in close collaboration with IRAP.

The CIS data sets available through the CAA interface are described in Dandouras et al. (2010). An updated report on the CIS calibration activities can be found on the CAA web page (current version: 1.4, see Dandouras et al., 2012).

The paper is organized as follows: in Sect. 2 the instrument and its specific parameters are presented. Section 3 discusses the HIA operation modes and data caveats. Section 4 provides details on the calibration tasks and illustrates the calibration methodology by two examples. The next section presents two statistical studies carried out for validating HIA's in-flight calibration. In Sect. 6 the results of HIA's in-flight calibration are summarized and plans to extend this activity are discussed.

\section{Instrument presentation}

Figure 1 presents the HIA operational principles. The instrument employs a $360^{\circ}$ angle imaging "top-hat" (Carlson et al., 1982) toroidal electrostatic analyzer (EA) and a fast detection system, based on MCP electron multipliers. The ions moving along different directions in the plane of the instrument entrance aperture (different polar angle in the upper panel of Fig. 1) are deflected and focused by the EA (middle panel) on the exit plane, where they are recorded by a system of position encoding discrete anodes (lower panel). Ion energies from $5 \mathrm{eV}$ to $32 \mathrm{keV}$ are sequentially measured by rapidly varying (sweeping), in logarithmically spaced steps, the voltage across the hemispherical EA plates. In the detection plane, the MCP plates are arranged in chevron-pair configuration in order to achieve a higher gain of secondary electrons emission. For a better detection efficiency, the ions are post-accelerated by a $\sim 2300-2500 \mathrm{~V}$ potential applied between the front of the MCP and a high-transparency grid located $\sim 1 \mathrm{~mm}$ above. The MCP gain can be checked by occasionally stepping this high voltage and by adjusting the discrimination level of the collecting charge amplifiers. Coverage in azimuthal angle is achieved by using the satellite spin.

To accommodate the large dynamic range of ion fluxes that occur in different regions sampled by Cluster, the entrance aperture consists of two narrow fans, each covering $180^{\circ}$ in polar angle and having sensitivities that differ by a factor of $\sim 25$. The high-sensitivity (HS or "high $G$ ") section (entrance aperture on the right in the upper panel of Fig. 1), selects ions with the appropriate energy per charge $(E / Q)$ and concentrates them on 16 anodes, $11.25^{\circ}$ each, located in the exit plane (on the left in the bottom panel of the figure). This section is designed for analyzing magnetospheric ions. Similarly, the low-sensitivity (LS or "low $g$ ") section (entrance aperture on the left in the upper panel of Fig. 1) is tuned for the detection of solar wind ions, i.e., for high ion fluxes with
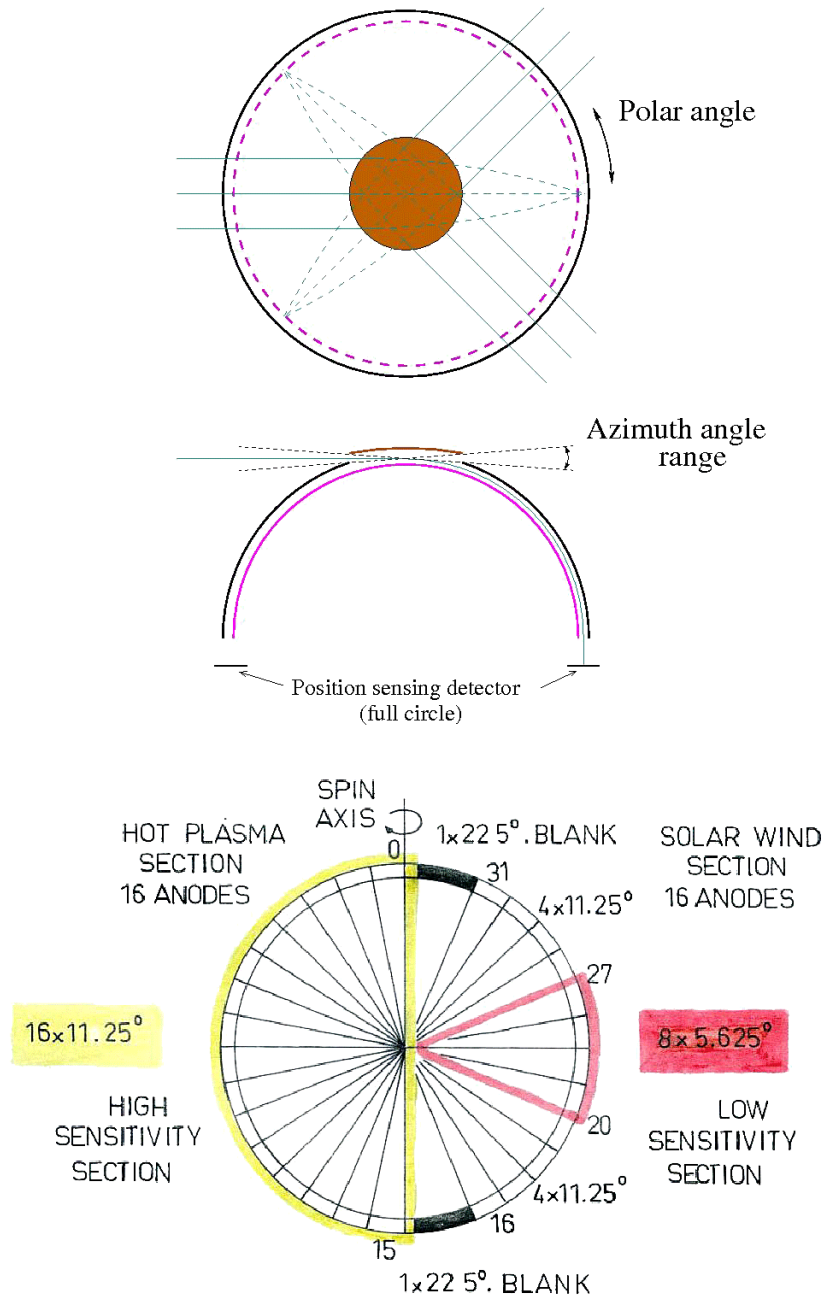

Fig. 1. The upper panels present the top view and cross-sectional view of the HIA instrument. In the bottom panel, the principles of HIA's anode sectoring are shown. In the upper and bottom panels of the figure, the spin axis is in the plane of the paper, along the vertical direction, while in the middle panel it points into the paper. See text for more details. Figure adapted from Klumpar et al. (2001) and Rème et al. (2001).

narrow energy and angular range. The required high angular resolution is achieved through the use of $8^{\circ} \times 5.625^{\circ}$ central anodes in the exit plane, the remaining 8 sectors having in principle $11.25^{\circ}$ resolution. In the solar wind mode, the HIA voltage sweep is truncated when the "high $G$ " section is facing the Sun, in order to avoid the solar wind detection and to protect the MCP lifetime. The two sections of the instrument can supply data simultaneously in the solar wind mode.

The HIA and CODIF sensors complement each other in terms of sensitivity, mass resolution, and detection efficiency. For CODIF, an additional time-of-flight (TOF) section is present, following the $E / Q$ selection by the EA, allowing thus the separation of ion species. However, for the HIA detector the efficiency is much larger, primarily because this 
Table 1. The HIA specific parameters according to Rème et al. (2001) and Dandouras and Barthe (2012).

\begin{tabular}{lrr}
\hline Energy range $(\mathrm{eV})$ & & $\sim 5-32 \times 10^{3}$ \\
$\begin{array}{l}\text { Energy resolution } \Delta E / E(\mathrm{FWHM}, \%) \\
\text { No. of } E \text { microsteps }\end{array}$ & 124 \\
\hline Time resolution (s) & 2-D & $62.5 \times 10^{-3}$ \\
& 3-D & 4 \\
\hline Angular resolution ( $\left.{ }^{\circ}\right)$ & LS & $\sim 5.6 \times 5.6$ \\
\hline Geom. factor $\left(\mathrm{cm}^{2} \mathrm{sr} \mathrm{keV} \mathrm{keV}^{-1}\right)$ & HS & $\sim 12.5 \times 5.6$ \\
\hline \multirow{2}{*}{ Dynamic range $\left(\mathrm{cm}^{2} \mathrm{~s} \mathrm{sr}\right)^{-1}$} & HS & $1.9 \times 10^{-4}$ \\
& LS & $10^{6}-2 \times 10^{-3}$ \\
\hline Lower limit density value $\left(\mathrm{cm}^{-3}\right)$ & HS & $10^{4}-2 \times 10^{8}$ \\
\hline
\end{tabular}

sensor has no TOF section. In addition, HIA provides a higher angular resolution (up to $5.6^{\circ} \times 5.6^{\circ}$, to be compared with $11.25^{\circ} \times 22.5^{\circ}$ for CODIF) and faster electronics, capable to handle higher count rates. This makes the HIA more suitable for the study of the solar wind environment. Table 1 summarizes the HIA specific parameters.

\section{HIA operation mode and data caveats}

There are 16 operating modes for the CIS instrument, which can be roughly grouped in 2 classes, i.e., "magnetospheric" and "solar wind" modes. In the magnetospheric modes the full energy-angle ranges are covered and the different data products are based on the counts accumulated on the "high $G$ " section. In the solar wind modes the plasma moments are based on data accumulated on the "low $g$ " section when this side is facing the solar wind direction. In each mode, HIA and CODIF share the telemetry bit rate allocated to CIS for transmitting scientific products (on-board computed moments, one-, two- and three-dimensional distributions and pitch-angle distributions) to the ground.

The HIA instrument involves extensive on-board data processing, including the computation of the moments of the velocity-distribution functions (density, bulk velocity vector, pressure tensor, and heat flux vector). The moments are transmitted to the ground every spin period, i.e., about $4 \mathrm{~s}$. The computation uses a table of efficiency coefficients (values dependent on the energy and angular sector $\theta$ ) based on the ground calibration performed before launch. Assuming the same energy dependence and symmetric anode efficiency evolution in time, the values of these moments are periodically adjusted on ground through the so called absolute calibration (see Sect. 4).

The transmission to the ground of the complete 3-D distribution function (i.e., at full angular and energy resolution) is not possible due to the limited telemetry rates allocated to the CIS experiment. For example, the nominal operation of HIA's HS section would require the transmission, every $4 \mathrm{~s}$, of a matrix having 62 (or 31) energy channels $\times 16$ elevation angles $\times 32$ azimuth angles $=31.744$ (or 15.872) elements. Therefore a reduced distribution function (particle counts typically binned in 31 energy channels and 88 angular directions) is computed on board and transmitted to the ground with a time resolution of multiple spin periods. Based on the reduced distribution function, the so-called "ground" plasma moments can be computed where, in principle, correction for the efficiency energy dependence and asymmetric anode efficiency evolution can be made.

Since in the solar wind spectrogram the $\mathrm{He}^{++}$trace is clearly separated, appearing as an ion beam at roughly twice the mean proton energy, the HIA is providing the plasma moments for this ion species as well. So far this data product has not been calibrated.

There are a number of data caveats of particular importance for the instrument calibration as well as for regular exploitation of HIA data. These aspects, briefly summarized below, are closely checked when selecting the calibration intervals. For a detailed discussion the reader is referred to the instrument web page http://cluster.irap.omp.eu/ (see also Rème et al., 2001; Dandouras and Barthe, 2012).

- The accuracy of computed moments is affected by the instrument's finite energy and angle resolution, and by its finite energy range. Also, a reliable plasma moment computation requires that enough counts (minimum $100)$ are accumulated over the spin period.

- Inappropriate operational mode adversely affects the data accuracy. For example, when HIA is in solar wind mode, while the measurements are taken in the magnetosphere, a large portion of the ion distribution is excluded. Similarly, when HIA is in the magnetospheric mode but measures in the solar wind, detector saturation may occur, leading to underestimated values for the plasma density.

- Due to the penetrating particles from the radiation belts, the HIA measures a high background around perigee passes. A similar effect may also occur during some intense solar particle events.

- The detection of low-energy ions may be affected by the spacecraft charging to a positive floating potential that repels these ions.

- On some occasions, instrument artifacts (wrong time tagging, sudden density drops, high-voltage discharges, wrong discriminator levels, etc.) may occur. These events are listed on the CIS Data Caveats list, available on the instrument's web page.

Since the beginning of the mission, the HIA sensors were operational only on C1 (Cluster 1) and C3. Since November 
2009, after almost 10 years of very good performance, the CIS experiment on board C3 is no longer operational. Also, since June 2011 the HIA operations on $\mathrm{C} 1$ are restricted to magnetospheric modes only, with the instrument switched into a safe stand-by mode when the satellite samples the solar wind region.

\section{The HIA in-flight calibration}

The HIA detection efficiency as a function of position (polar angle $\theta$ ) and particle energy $E$ is given by the formula (see Bosqued, 2000, reporting on HIA's ground calibration)

$$
\operatorname{Eff}(\theta, E)^{-1}=\operatorname{Norm} \_\theta \cdot \operatorname{Cheff}(\theta) \cdot \frac{A \cdot E+B}{T_{0}+T_{1} \cdot E_{\mathrm{t}}+T_{2} \cdot E_{\mathrm{t}}^{2}}
$$

The first part of the RHS describes the position (anode)dependent efficiency, with Norm_ $\theta$ designating the anode normalization coefficients (one for each sensitivity side) and $\operatorname{Cheff}(\theta)$ the relative anode-dependent efficiency coefficients. The second part of RHS describes the efficiency energy dependence, with $A, B$ and $T_{0,1,2}$ being the calibration coefficients and $E_{\mathrm{t}}=E+E_{\mathrm{g}}$ the total energy (sum of the particle energy $E$ and the MCP - grid acceleration energy $\left.E_{\mathrm{g}}\right)$ employed for describing the MCP energy-dependent efficiency. In total there are $2+2 \cdot 16+2 \cdot 2+3=41$ (39 independent) efficiency calibration coefficients for each validity period and spacecraft. Their values are specified in the calibration files that are constantly provided as the mission progresses.

The efficiency coefficients of the HIA instruments have been determined on ground through extensive preflight calibrations at IRAP vacuum-test facilities in Toulouse. Using ion beams of energies from a few $10 \mathrm{eV}$ up to $30 \mathrm{keV}$, detailed studies of MCPs gain levels, MCP matching, and angular-energy resolution for each sector (each $\theta$ ) were performed. Based on these tests, a table of efficiency coefficients is stored on-board in the nonvolatile memory and used by the processing software to compute the on-board moments from the full angular and energy resolution 3-D ion-distribution function.

However, the detector efficiencies change with time due to various reasons presented in Sect. 1. Therefore, as the missions progresses, the on-board calculations are based on outof-date/in-accurate efficiencies. These unavoidable changes with time of the channel-plate detectors require continuous in-flight calibration. Also, the MCP high voltage is periodically increased by ground commands to compensate for the MCP gain fatigue. Since the procedure has a direct impact on detector efficiency, an in-flight calibration is subsequently required. So far, this operation has been performed five times (see Fig. 8).

The standard procedure for HIA's in-flight calibration, called the absolute calibration, relies on comparing HIA's ion number density with the electron number density pro- vided by the WHISPER (Décréau et al., 2001) experiment on board Cluster. While the HIA detects individual particles to measure the ion-distribution function, WHISPER is based on a different method to determine the plasma density, i.e., by analyzing, both actively and passively, the electric signals in the neighboring plasma. In active mode, WHISPER measures the total electron density, while in passive mode it provides a survey of natural emissions from about 2 to $80 \mathrm{kHz}$, which covers the electron plasma resonance frequency.

The following are a number of assumptions involved in the absolute calibration procedure.

- There is a symmetric anode-dependent efficiency evolution with time, and therefore the relative anodedependent efficiency coefficients $\operatorname{Cheff}(\theta)$, determined in the preflight tests, have not changed.

- The coefficients $A, B$ and $T_{0,1,2}$ describing the efficiency energy dependence do not change as well and assume the values determined in the preflight tests.

- The WHISPER data are well-calibrated and free of errors (at least in the statistical sense). The traces in the WHISPER spectrograms are correctly assigned to some characteristic frequencies in plasma, from where electron density can be inferred. In these circumstances, the WHISPER data can be taken as reference.

The first two assumptions greatly simplify the calibration task, basically implying that only two coefficients (one for each sensitivity side) are needed to correct the HIA efficiency. It also means that the on-board moments are accurate up to a multiplication factor determined through calibration, thus allowing to take advantage of the HIA's highest temporal, directional and energy resolution. Indeed, e.g., in magnetospheric modes, the on-board moments are computed every spin period based on uncompressed data accumulated in 32 energy channels and 16 elevation $\times 32$ azimuth solid angles (Di Lelis and Formisano, 2000), whereas the groundcomputed moments are based on the reduced distribution function transmitted to the ground, having typically 31 energy $\times 88$ solid angle bins and poorer time resolution.

Figure 2, based on $\mathrm{C} 1$ data from 17 October 2007, presents the individual anode response of the HIA high-sensitivity section in the plasma-sheet environment, where the plasmadistribution function is expected to be highly isotropic. Each of the panels corresponds to one sector in elevation angle ( $\theta$ angle) for the arriving particles. The relatively homogeneous response from all eight angular sectors qualitatively supports the assumption of a symmetric anode-dependent efficiency evolution with time. The same situation is observed for the HIA instrument on $\mathrm{C} 3$ as well. Note that there are only 8 panels, although according to bottom panel in Fig. 1 there should be 16 angular sectors for the HS side of the HIA. This is because the distribution function sent to the ground has 


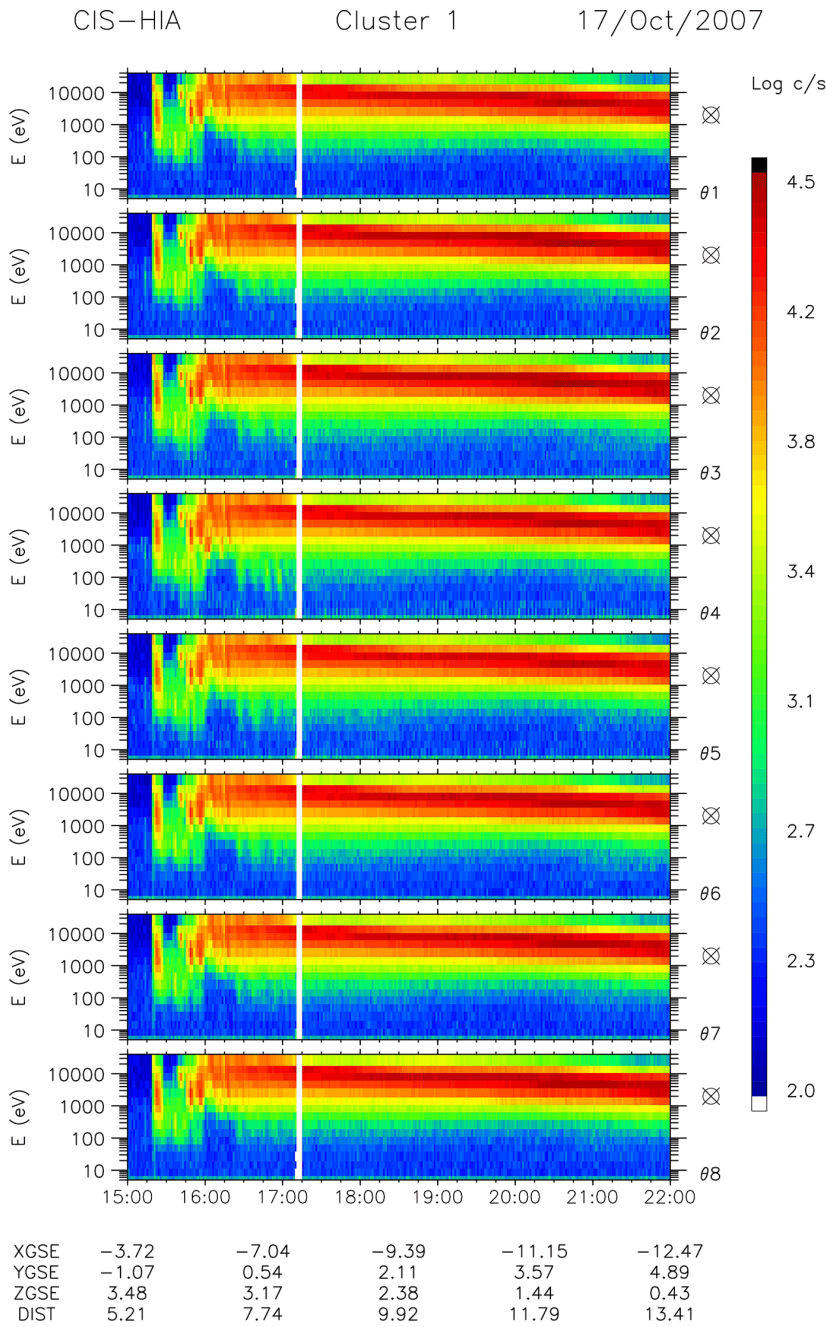

Fig. 2. Individual anode response of HIA's high-sensitivity section in the plasma sheet, an environment where the plasma-distribution function is highly isotropic. Only $\mathrm{C} 1$ data are shown. Each of the panels corresponds to one sector in elevation angle for the arriving particles.

been reduced by the on-board processing software in order to comply with the limited capacity of the telemetry; in that process the counts registered by individual anodes are binned in eight angular sectors.

Regarding the second assumption, i.e., constancy of the coefficients describing the efficiency energy dependence, there are some indications that this might not be completely valid (see e.g., the discussion about the HIA measurements in the plasma-sheet environment in Sect. 6) but so far no careful study addressing this problem has been carried out. However, it seems that a change in the efficiency energy dependence is a second-order effect, at least in the plasma environments where the two HIA sides are calibrated (see the statistical studies presented in Sect. 5).
One particular aspect that might be of concern is the role of ion composition in the HIA-WHISPER data comparison. The prevalent minor ions in solar wind and magnetosheath plasma (the environments where the two HIA sides are calibrated; see next sections) are the $\alpha$ particles. If one considers a mixture of protons (number density $N_{\mathrm{p}}$, mass $m_{\mathrm{p}}$, and electric charge $q_{\mathrm{p}}$ ) and $\alpha$ particles (with corresponding parameters $N_{\alpha}, m_{\alpha}$, and $q_{\alpha}$ ), then for a detector like HIA, unable to discriminate between the ion species, the number density reported by the instrument will be (see e.g., Paschmann et al., 1998) $N_{\mathrm{HIA}}=N_{\mathrm{p}}+\sqrt{m_{\mathrm{p}} / m_{\alpha}} N_{\alpha}=N_{\mathrm{p}}+N_{\alpha} / 2$. However, the WHISPER instrument will report a number density of $N_{\mathrm{WHI}}=N_{\mathrm{p}}+\left(q_{\alpha} / q_{\mathrm{p}}\right) N_{\alpha}=N_{\mathrm{p}}+2 N_{\alpha}$. Typically, the $\alpha$ particles' abundance in the solar wind and magnetosheath plasma is around few percents of the proton number density. Therefore the discrepancy between the readings of the two instruments is of the same magnitude and consequently can be neglected in the first approximation. In addition, the procedure used to select the final set of calibration intervals (see Sect. 4.1) tends to exclude intervals with lower (than expected) values of the $N_{\mathrm{HIA}} / N_{\mathrm{WHI}}$ ratio.

In the case of the HIA operating in solar wind mode, where the protons and $\alpha$ particles are clearly separated in the energy/charge channels, the on-board software automatically computes the plasma moments separately for the two ion species. Therefore here one compares $N_{\mathrm{HIA}}=N_{\mathrm{p}}$ with $N_{\mathrm{WHI}}=N_{\mathrm{p}}+2 N_{\alpha}$. However, the clear separation of the $\mathrm{He}^{++}$trace in the energy spectrogram allows us to select from the beginning intervals with low presence of $\alpha$, as will be described in Sect. 4.2.

\subsection{Calibration of HIA's high-sensitivity section}

For calibrating HIA's high-sensitivity section, magnetosheath (MS) intervals are used since the characteristic values of the plasma parameters in that environment (like density, temperature, and energy spectrum within the energy domain covered by HIA) allow for an optimum instrument performance.

Each part of the year in which Cluster samples the MS environment, i.e., roughly between November and June the following year, is analyzed to obtain one set of calibration coefficients. The number of values in the set depends on the efficiency evolution. Typically, two values, each obtained by combining data from several intervals, are inferred. Nevertheless, when an increase in the MCP HV is commanded from ground, a sudden increase followed by a relatively rapid adjustment in efficiency is expected, which requires the determination of additional values. The last value in the set is assumed to be valid until the beginning of the next MS season.

Intervals for calibration are carefully selected to meet several criteria listed below.

- The HIA energy spectrogram suggests that, presumably, the vast majority of ions are detected, i.e., no 
indication of a significant ion population below or above the detector energy range exists.

- The evolution of HIA's density is regular, useful for revealing potential instrument artifacts. This condition is also important because the HIA and WHISPER instruments could provide different values when a steep boundary is encountered (due to Larmor radius effects). It is desirable as well to select intervals where the density values span over a wide range, for a better comparison.

- Preferably the same intervals for both C1 and C3 are used to allow for an interspacecraft calibration and to detect potential instrument artifacts.

- Intervals are not on the Data Caveat list (see Sect. 3).

For the selected intervals, the WHISPER density data are requested from the instrument team, which either decides to regenerate it for the purpose of HIA calibration or to revalidate the already available CAA data set.

Figure 3 illustrates one example of an interval selected for the HIA-WHISPER density comparison. The four panels at the top represent the type of plot routinely produced for the identification of calibration intervals. First, the HIA energy spectrograms in three ranges, i.e., above $2 \mathrm{keV}$, the entire energy range, and below $100 \mathrm{eV}$, are shown in order to identify intervals that better comply with the requirement that virtually all particles are detected by the instrument. In the fourth panel the HIA (red) and WHISPER (black) raw density data are shown. Here a few sudden HIA density drops can be seen; these signatures are not present in the WHISPER data and are interpreted as instrument artifacts. The next panel compares the two densities after the data processing has been performed, which includes the removal of instrument artifacts, discarding of short intervals with rapid variation in density, data filtering, averaging and interpolation, etc. The sixth panel checks the plasma gyrotropy as measured by the quantity $\left(p_{\perp 2}-p_{\perp 1}\right) /\left[\left(p_{\perp 2}+p_{\perp 1}\right) / 2\right]$, with $p_{\perp 1}$ and $p_{\perp 2}$ being the plasma thermal pressure along two orthogonal directions in the plane perpendicular to the magnetic field. The relatively small deviations from gyrotropy, e.g., around or below $5 \%$, provides supporting evidence of the HIA's symmetric anode response for this interval. The bottom panel shows the $N_{\text {HIA }} / N_{\text {WHI }}$ density ratio (blue) and its average value for this interval (magenta straight line).

The result of comparison can also be shown in the form of a HIA vs. WHISPER density plot, presented in Fig. 4, where the left panel refers to the event described above. The points are clearly scattered along the regression line (in red) forced to cross the origin; its slope is used to estimate the calibration factor inferred from this interval. The average value of several calibration factors, obtained from different intervals, is then employed to update the calibration files used in processing the HIA data.

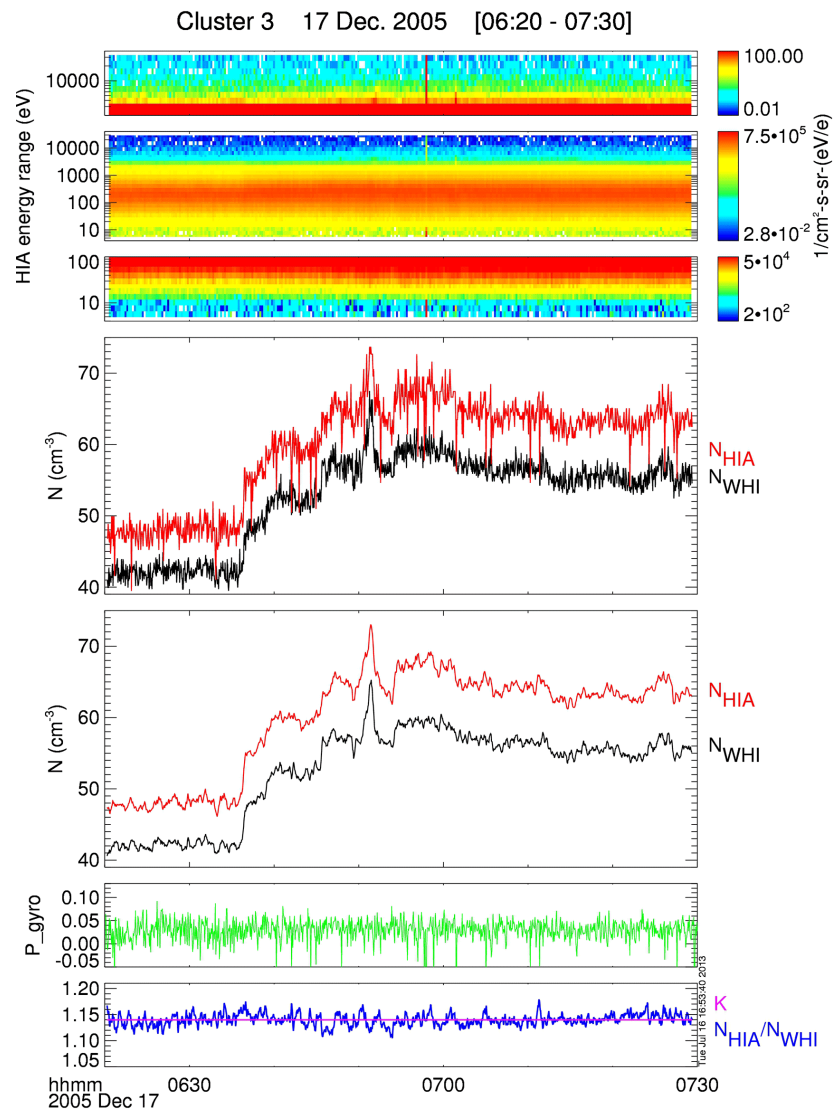

Fig. 3. Calibration of HIA's HS section. The top panels show ion energy spectrograms in three ranges, i.e., above $2 \mathrm{keV}$, the entire HIA energy range, and below $100 \mathrm{eV}$. The next panel presents HIA (red) and WHISPER (black) raw density data while in the fifth panel, the data from the two instruments are processed. The sixth panel presents the deviation in gyrotropy, based on the relative differences between the perpendicular pressure components. The bottom panel shows the ratio between the two densities (blue) and its average value $K$ (magenta straight line). More explanations are provided in the text.

In spite of the careful selection, it can happen that the HIA-WHISPER data comparison brings inconsistent results on some intervals. Typically a lower (than expected) value of the $N_{\mathrm{HIA}} / N_{\mathrm{WHI}}$ ratio is attributed to the presence of plasma population outside the HIA detection range, to the spacecraft charging or to events with relatively high abundance of $\alpha$ particles. Therefore, the final set of intervals to be used in the calibration is established after an additional interspacecraft comparison and/or checking the data provided by other instruments like CODIF, ASPOC, and EFW (spacecraft potential).

\subsection{Calibration of HIA's LS section}

With some specific differences, the calibration of the HIA low-sensitivity section follows a similar procedure. One uses 

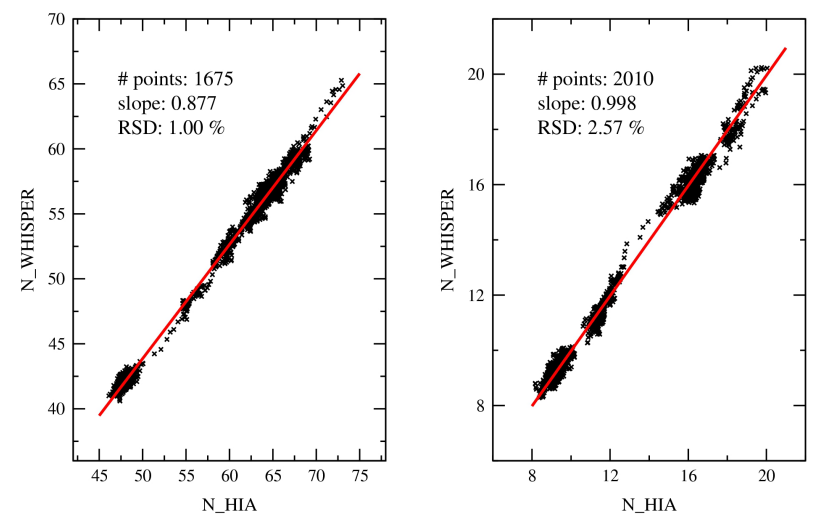

Fig. 4. HIA vs. WHISPER density comparison for the events presented in Fig. 3 (left) and in Fig. 5 (right), employed to calibrate the HIA HS and LS sections, respectively. The slope of the regression line crossing the origin (in red) is used to estimate the calibration factor for the corresponding interval.

solar wind (SW) data and compares the HIA and WHISPER density on carefully selected intervals.

The selection is based on the type of plot presented in Fig. 5. The top panel shows HIA (red) and WHISPER (black) raw density data, A number of sudden HIA density drops (instrument artifacts) can be seen here as well. In the second panel the energy spectrogram based on data accumulated on the LS section is shown. For the purpose of calibration, it is desirable to select events with low presence of $\alpha$ particles, (seen in the second panel as the faint green line, at around twice the peak proton energy). Since an accurate calibration of the LS section requires no significant counts accumulated on the HS section (blocked when facing the SW direction, see Sect. 2), the third and the fourth panel present the plasma density and the energy spectrogram based on data accumulated on this side. The bottom panel compares the LS (red) and HS (black) count rates.

After the selection of SW intervals suitable for calibration, the raw HIA and WHISPER data are processed in a manner similar to that described in the previous section. For the event presented above, the result of the comparison is shown in the right panel of Fig. 4 in the form of a HIA vs. WHISPER density plot. The points are aligned along the regression line (in red) forced to cross the origin; its slope is taken as the value of the calibration factor corresponding to this interval. By averaging several calibration factors, inferred from different intervals, a value is obtained that will be used to update the calibration files.

\section{Statistic comparison between HIA and WHISPER data}

To validate the results of calibration methodology presented in Sect. 4, two statistical studies have been carried out, each based on data provided by one HIA sensitivity side.

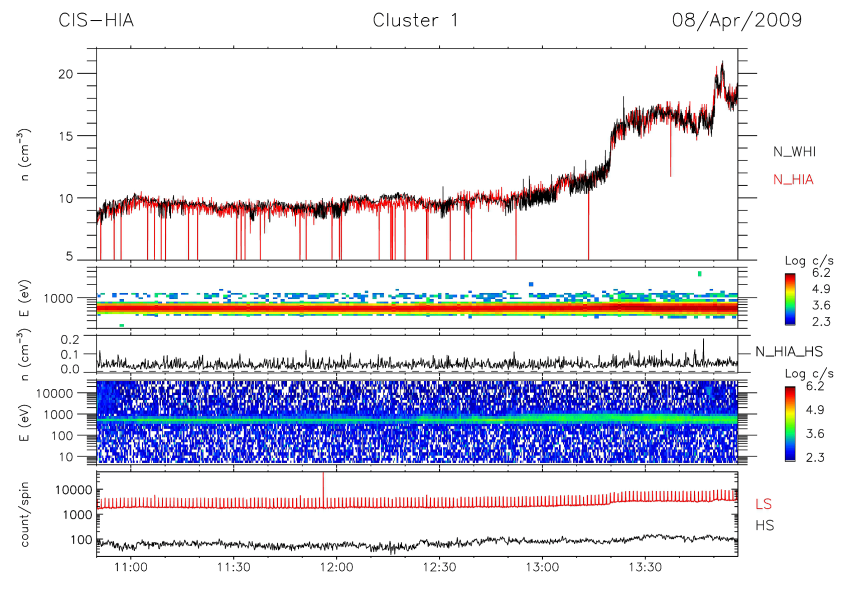

Fig. 5. Standard plot used for identification of suitable HIA LS calibration intervals. From top to bottom, the individual panels present HIA (red) and WHISPER (black) raw density data, the energy spectrogram provided by the LS section, the number density based on data collected by the HS section, the energy spectrogram provided by the HS section, and the number of counts detected by LS (red) and HS (black) sections.

All MS and SW intervals observed in Cluster 1 data between 15 December 2006 and 15 February 2007 have been analyzed in order to compare calibrated HIA and WHISPER density data. Stable detector efficiency is expected in that period since the previous MCP high-voltage increase occurred in January 2006, the next one being performed on 16 February 2007 , i.e., just after the chosen interval. Using selection criteria similar to those presented in Sect. 4, a total of 54 MS intervals sampled by the HIA HS section have been identified, comprising around $55 \mathrm{~h}$ of data. For the LS section, $64 \mathrm{SW}$ intervals have been identified, covering around $96 \mathrm{~h}$ of data.

The results of the analysis are presented in Fig. 6 for the HS section and in Fig. 7 for the LS section. In the former case, the regression line (in red) forced to cross the origin corresponds to a proportionality factor of $\sim 1.01$; the relative standard deviation (RSD) of calibrated HIA data with respect to WHISPER data is $8.3 \%$. For the LS side, the regression line corresponds to a proportionality factor of $\sim 1.02$, with the RSD of points of $5.7 \%$.

The following are a number of conclusions that can be drawn from these comparisons.

- The dependence between $N_{\mathrm{HIA}}$ and $N_{\mathrm{WHI}}$ is linear. The proportionality factor is close to the ideal value of 1 , a result that validates the calibration methodology outlined in Sect. 4.

- At least in the two plasma environments used for HIA's in-flight calibration, the assumptions of symmetric anode-dependent efficiency evolution and constancy of the coefficients describing the efficiency 


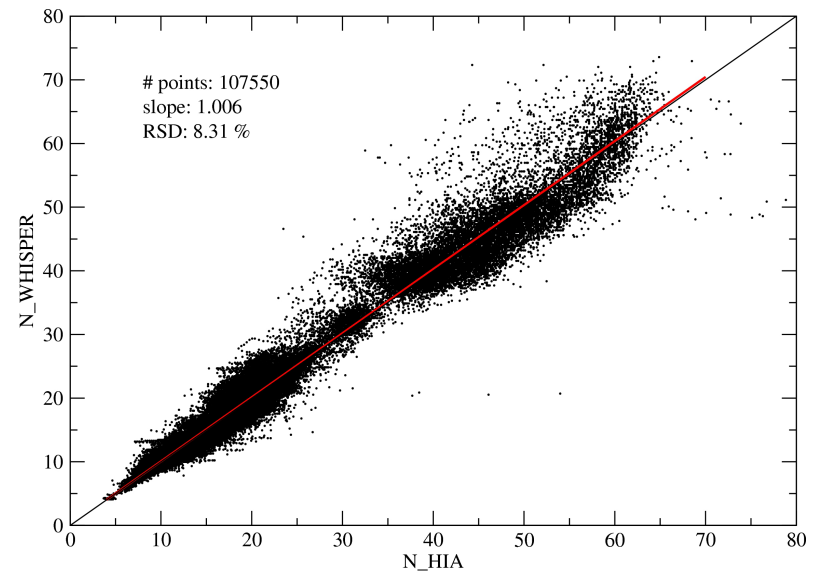

Fig. 6. Comparison between calibrated HIA and WHISPER data on Cluster 1. All magnetosheath intervals sampled by the HIA HS side between 15 December 2006 and 15 February 2007 were selected for comparison using selection criteria similar to those presented in Sect. 4. The regression line crossing the origin is shown in red.

energy dependence are verified in the statistical sense, any deviation being of a second-order importance.

- Since the instrument's artifacts have not been removed before comparison, the results indicate that they have no significant influence on the data, in a statistical sense.

\section{Summary and future work}

The first two panels in Fig. 8, showing the evolution of HS and LS detection efficiency in the course of the mission, summarize the results of HIA's in-flight calibration activity. Our comments below will refer mainly to the period starting from October 2005, subject to the calibration methodology presented in this paper.

The top and middle panels in Fig. 8 refer to the HS and LS sections, respectively. The detector efficiencies shown, obtained by comparing the HIA and WHISPER data densities, are relative to the beginning of the mission. Black lines present the evolution of $\mathrm{C} 1$ while the red lines the evolution for C3. Each value of the efficiency is shown by a horizontal segment, with the length indicating its validity period. There are no HIA data on C3 after November 2009 as well as on the C1 LS side after June 2011 (see the last paragraph of Sect. 3).

It is worth noting that the HIA detection efficiency stayed at a reasonable level in the course of the mission. Taking, for example, the values corresponding to 2009, the relative efficiency on $\mathrm{C} 1$ was around 1 for the HS side and 0.93 for the LS side, whereas in the case of $\mathrm{C} 3$, the relative efficiency was around 1.25 for the HS side and 1.38 for the LS side.

The high voltage applied to the MCPs is presented in the bottom panel; the vertical dashed lines indicate the dates

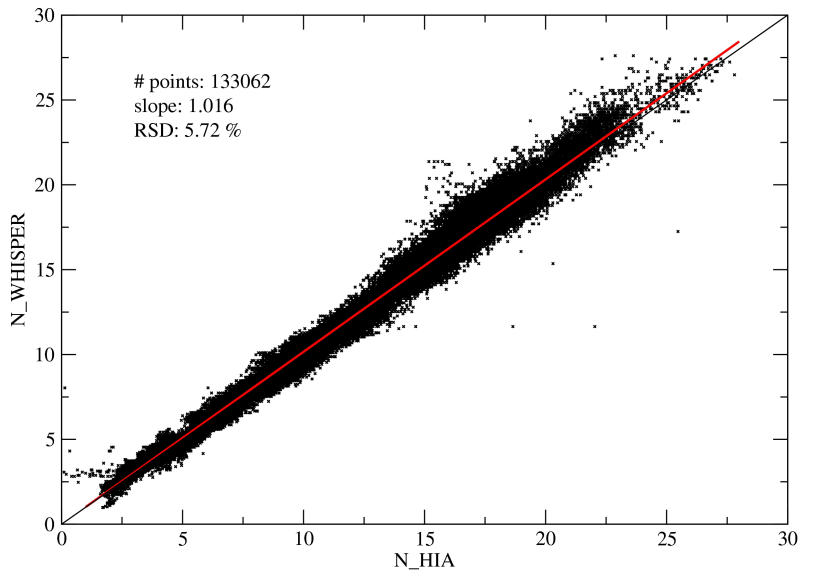

Fig. 7. Comparison between calibrated HIA and WHISPER data on Cluster 1. All solar wind intervals sampled by the HIA LS side between 15 December 2006 and 15 February 2007 were selected for comparison using selection criteria similar to those presented in Sect. 4. The regression line crossing the origin is shown in red.

when this HIA operating parameter was raised by ground commands for the purpose of increasing the detection efficiency. With some exceptions (e.g., see the LS side after the last change, on 17 February 2007) such an increase has been noticed. Note also that sometimes the efficiency has slightly increased without raising the MCP HV, like at the end of 2007. This unexpected behavior has been observed on both spacecraft and on both sensitivities, i.e., both on MS and SW intervals, which argues for a real effect. An MCP efficiency recovery has also been reported by the PEACE (Plasma Electron And Current Experiment) team in that period.

The HIA in-flight calibration is a complex task that requires considerable effort. This activity will continue and expand in order to ensure the highest data accuracy. Below we present a list of topics to be addressed in the future.

- Applying the calibration methodology described in this paper to data provided by HIA in the first years of the Cluster mission, i.e., before October 2005.

- Investigating more closely whether or not the anodedependent efficiency factors evolved symmetrically in the course of the mission. Although Fig. 2 indicates a relatively homogeneous response from all HIA angular sectors, no quantitative assessment of this assumption was made so far and only few data intervals have been qualitatively evaluated. In the case of CODIF such an investigation is regularly performed, bringing significant improvements to data quality.

- Investigating possible changes in the efficiency energy dependence in the course of the mission.

- Calibrating the $\mathrm{He}^{++}$data provided by HIA in the SW. 


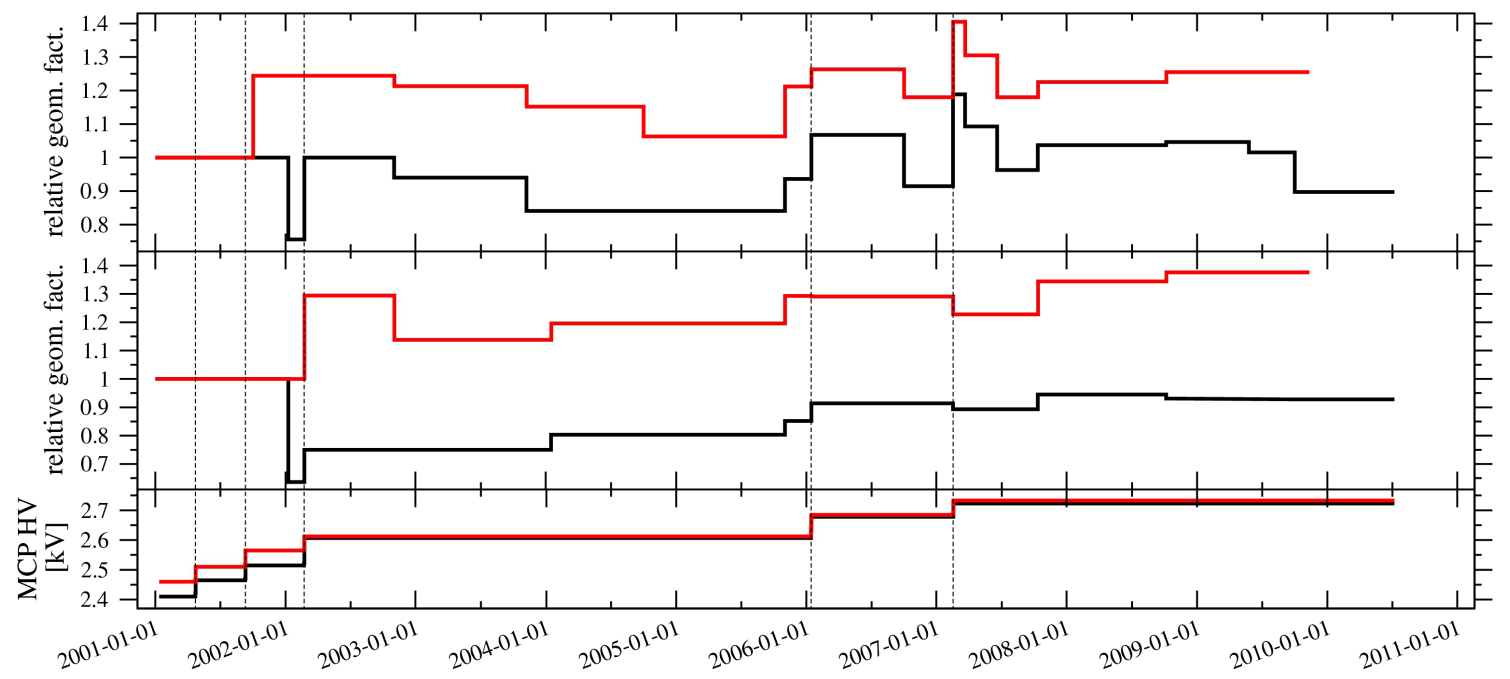

Fig. 8. Evolution of the HIA HS and LS efficiency (top and middle panels, respectively), and of the high voltage applied to the MCPs (bottom panel). Black lines refer to $\mathrm{C} 1$ and red lines to C3. The time axis spans from 1 January 2001 (around the start of Cluster's operational phase) to the beginning of 2011. The dates of MCP HV increase are also shown by vertical dashed lines.

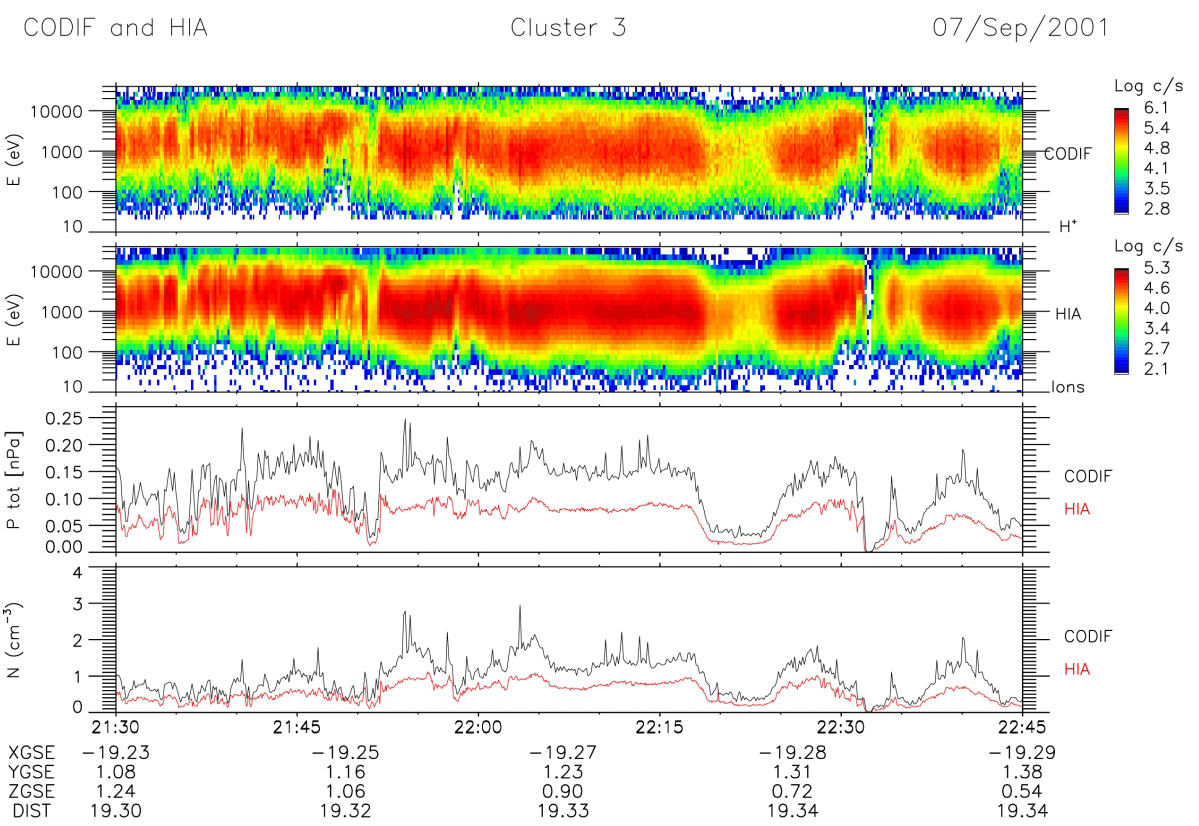

Fig. 9. CODIF and HIA data comparison in the plasma-sheet region. From top to bottom, the first two panels show the differential energy spectrogram from CODIF and HIA, respectively. The next two panels show the plasma pressure and plasma density as provided by CODIF (black line) and HIA (red line).

Related to the third item above, there is evidence suggesting the need for correction of the efficiency energy dependence. Figure 9 shows a comparison between HIA and CODIF data in the plasma-sheet environment on 7 September 2001. The energy spectrograms (CODIF first panel and HIA the second panel) support the idea that most of the particles are detected by the two instruments. However, the plasma pressure and density (next panels) are different, with HIA (red lines) providing values around two times lower that
CODIF (black lines). The two instruments are calibrated and there is convincing evidence that CODIF provides the correct measurements.

The situation presented in Fig. 9 is typical for the plasmasheet. The HIA-CODIF data discrepancy in this environment could be explained by changes in the efficiency energy dependence, since the average plasma energy in the plasma-sheet is higher than in the magnetosheath, where the instrument was calibrated. It means that a correction 
that results in lower efficiency for the high-energy channels would provide a plasma density correction in the right direction (i.e., an increase in density and, mostly, in pressure). The same type of correction has been suggested by the instrument response to the penetrating radiation during perigee passes (see Ganushkina et al., 2011).

Acknowledgements. The authors would like to thank the WHISPER team (Principal Investigator, J. L. Rauch; former Principal Investigators, P. M. .E. Décréau and Jean Gabriel Trotignon) for their effort to ensure reliable electron density data for the HIA calibration.

The calibration-routine package is based on the $\mathrm{cl}$ (credit to E. Penou) and CCAT (credit to J. McFadden, P. Schroeder and C. Mouikis) programs for CIS data analysis.

The work has been supported through the project VALS (contract 45/19.11.2012) of the Romanian Space Agency STAR program, and by the OTKA grant K75640 of the Hungarian Scientific Research Fund.

Edited by: H. Laakso

\section{References}

Bosqued, J. M.: Cluster-2: CIS-2 Instrument: Summary of Calibration Tables, CESR report, Toulouse, 2000.

Carlson, C. W., Curtis, D. W., Paschmann, G., and Michel, W.: An instrument for rapidly measuring plasma distribution functions with high resolution, Adv. Space Res., 2, 67-70, doi:10.1016/0273-1177(82)90151-X, 1982.

Dandouras, I. and Barthe, A.: User Guide to the CIS measurements in the Cluster Active Archive (CAA), http://caa.estec.esa.int/caa/ ug_cr_icd.xml (last access: 4 July 2013), 2012.

Dandouras, I., Barthe, A., Kistler, L. M., and Blagau, A.: Calibration Report of the CIS measurements in the Cluster Active Archive (CAA), http://caa.estec.esa.int/caa/ugcricd.xml (last access: 15 April 2014), 2012.

Dandouras, I., Barthe, A., Penou, E., Brunato, S., Rème, H., Kistler, L. M., Bavassano-Cattaneo, M. B., and Blagau, A.: Cluster Ion Spectrometry (CIS) Data in the Cluster Active Archive (CAA), in: The Cluster Active Archive, Studying the Earth's Space Plasma Environment, edited by: Laakso, H., Taylor, M., and Escoubet, C. P., 51-72, Springer, Berlin, 2010.

Décréau, P. M. E., Fergeau, P., Krasnoselskikh, V., Le Guirriec, E., Lévêque, M., Martin, Ph., Randriamboarison, O., Rauch, J. L., Sené, F. X., Séran, H. C., Trotignon, J. G., Canu, P., Cornilleau, N., de Féraudy, H., Alleyne, H., Yearby, K., Mögensen, P. B., Gustafsson, G., André, M., Gurnett, D. C., Darrouzet, F., Lemaire, J., Harvey, C. C., Travnicek, P., and Whisper experimenters (Table 1): Early results from the Whisper instrument on Cluster: an overview, Ann. Geophys., 19, 1241-1258, doi:10.5194/angeo-19-1241-2001, 2001.
Di Lelis, A. M. and Formisano, V.: Cluster CIS-2 Instrument, FM Normal Operation Software, CNR IFSI report, Frascati, 2000.

Ganushkina, N. Y., Dandouras, I., Shprits, Y. Y., and Cao, J.: Locations of boundaries of outer and inner radiation belts as observed by Cluster and Double Star, J. Geophys. Res.-Space, 116, A09234, doi:10.1029/2010JA016376, 2011.

Klumpar, D. M., Möbius, E., Kistler, L. M., Popecki, M., Hertzberg, E., Crocker, K., Granoff, M., Tang, L., Carlson, C. W., McFadden, J., Klecker, B., Eberl, F., Künneth, E., Kästle, H., Ertl, M., Peterson, W. K., Shelly, E. G., and Hovestadt, D.: The Time-ofFlight Energy, Angle, Mass Spectrograph (Teams) Experiment for Fast, Space Sci. Rev., 98, 197-219, 2001.

Paschmann, G., Fazakerley, A. N., and Schwartz, S. J.: Moments of plasma velocity distributions, in: Analysis Methods for MultiSpacecraft Data, edited by Paschmann, G. and Daly, P. W., ISSI Scientific Reports, 125-158, ESA Publications Division, Noordwijk, 1998.

Prince, R. H. and Cross, J. A.: Gain Fatigue Mechanism in Channel Electron Multipliers, Rev. Sci. Instrum., 42, 66-71, doi:10.1063/1.1684879, 1971.

Rème, H., Aoustin, C., Bosqued, J. M., Dandouras, I., Lavraud, B., Sauvaud, J. A., Barthe, A., Bouyssou, J., Camus, Th., Coeur-Joly, O., Cros, A., Cuvilo, J., Ducay, F., Garbarowitz, Y., Medale, J. L., Penou, E., Perrier, H., Romefort, D., Rouzaud, J., Vallat, C., Alcaydé, D., Jacquey, C., Mazelle, C., d’Uston, C., Möbius, E., Kistler, L. M., Crocker, K., Granoff, M., Mouikis, C., Popecki, M., Vosbury, M., Klecker, B., Hovestadt, D., Kucharek, H., Kuenneth, E., Paschmann, G., Scholer, M., Sckopke, N., Seidenschwang, E., Carlson, C. W., Curtis, D. W., Ingraham, C., Lin, R P., McFadden, J. P., Parks, G. K., Phan, T., Formisano, V., Amata, E., Bavassano-Cattaneo, M. B., Baldetti, P., Bruno, R., Chionchio, G., Di Lellis, A., Marcucci, M. F., Pallocchia, G., Korth, A., Daly, P. W., Graeve, B., Rosenbauer, H., Vasyliunas, V., McCarthy, M., Wilber, M., Eliasson, L., Lundin, R., Olsen, S., Shelley, E. G., Fuselier, S., Ghielmetti, A. G., Lennartsson, W., Escoubet, C. P., Balsiger, H., Friedel, R., Cao, J.-B., Kovrazhkin, R. A., Papamastorakis, I., Pellat, R., Scudder, J., and Sonnerup, B.: First multispacecraft ion measurements in and near the Earth's magnetosphere with the identical Cluster ion spectrometry (CIS) experiment, Ann. Geophys., 19, 1303-1354, doi:10.5194/angeo19-1303-2001, 2001.

Vallat, C.: Evaluation des performances en vol des détecteurs de l'expérience "Cluster Ion Spectrometry", CNRS report, Toulouse, 2001. 